\title{
Editorial
}

\section{Seeking a new 'normal' in the Canadian food environment}

\author{
Lana Vanderlee, PhD, Guest Editor (1); Erin P. Hobin, PhD, RD (2)
}

Our food environment, which includes physical and social surroundings, has 'normalized' unhealthy eating behaviours in Canada. The ubiquity of unhealthy, energy dense, nutrient poor foods in the settings in which we go about our daily lives has made these foods the norm in our dietary patterns. This ease of access and affordability, coupled with high palatability and heavy marketing of less healthy food choices has established food preferences that are not in alignment with population-level dietary goals, and the result is poor diet quality among the vast majority of Canadians. ${ }^{1}$ But what if 'healthy' became the new norm that is expected, demanded, and preferred from the population?

Many food preferences are malleable, and can be shaped over time by external cues. ${ }^{2,3}$ As such, experts have identified a range of policy options that can contribute to normalizing and increasing preferences for healthier food options. ${ }^{2}$ Some of these policy options have been explored in previous special issues on the Canadian food environment in this journal, including marketing and advertising practices, food formulation that is lower in salt, sugar and fat, and physical access to more healthy and fewer less healthy food outlets. In the last of our special issues on the food environment, the showcased articles have implications for additional policy options targeting food environments that aim to influence food access, availability and perceptions of the food system which have implications for shaping food choices and food preferences. Realistically, food environments are unlikely to change independently without public demand prioritizing nutrient-rich foods across settings; therefore, it is likely that food environments and food preferences will shift in a healthier direction somewhat concurrently, as they are mutually reinforcing. Increasing the availability of nutritious foods in our everyday lives will 'normalize' their presence and decrease barriers that discourage healthy choices, thereby shaping preferences and demand. As the public becomes more aware and informed of the importance of healthy food choices, they will increasingly demand that these choices are more readily available and affordable, and our food system will need to respond.

This issue includes a commentary by the Office of the Chief Dental Officer of Canada that describes the impact of the current food environment on oral health in Canada. ${ }^{4}$ The piece describes the normalization of food and drinks high in added sugar (with a focus on sugar-sweetened beverages) in the diet of children, youth and young adults in Canada, and the negative impact on oral health. The article describes how creating supportive food environments that make healthier choices more available, affordable, and accessible (and therefore, more 'normal') has significant potential to decrease the burden of poor oral health, in addition to a variety of other non-communicable diseases, and reinforces the importance of oral health experts as contributors to the food environment conversation.

Changing the foods that are consistently available to consumers across settings can substantially influence food choices, as demonstrated in the paper by Raine and colleagues. ${ }^{5}$ In particular, settings where children frequent, such as schools and recreation centres play an integral role in establishing food preferences by modelling eating behaviours, and thereby shaping social and cultural norms among this impressionable age group. Health care and other government-funded facilities serve as examples for the general public with regard to what foods they believe are acceptable to be served, as ostensibly these are the very institutions that have significant investment in population health and health care costs. The article summarizes consensus recommendations developed by thought leaders from across the country for procuring and providing healthy foods in public sector settings, and highlights the critical roles that multiple stakeholders are required to perform in successful implementation of healthy food procurement policies on a large scale.

The article by McIsaac et al. provides reflection on what the implementation of a procurement policy looks like at the local level, in this case in recreation and sport facilities. ${ }^{6}$ The article demonstrates ingrained social norms and values with regard to the foods that are expected to be available in recreation and sports settings in Canada and consumer demand for less healthy products in these environments. It also identifies values regarding the perceived appropriateness of public venues controlling consumer food choices, and the concept of individual responsibility for food choices related to obesity. The findings highlight the shifts in cultural norms and values related to consumer demand and personal responsibility for food choices that will be required for healthy procurement policies to be implemented successfully, and how these shifts in consumer expectations may occur simultaneously as these types of policies become more commonplace across settings.

The status report by Coleman and colleagues describes a novel approach to healthy food procurement among community 
organizations with the development of the FoodReach initiative, a program that brings together small organizations to increase purchasing power for healthy, fresh food products. ${ }^{7}$ This approach is a valuable example of how to successfully implement healthy procurement policies in small organizations. The creation of a knowledge exchange portal will also help with cross-learning from policy and program experience, which was a recommendation stemming from the expert consensus by Raine and colleagues. Finally, the FoodReach program provides an opportunity for community groups to model healthy eating behaviours in a variety of community programs, which range from school nutrition programs to child care centres, to resource centres for those experiencing homelessness.

The final article in this special issue by Martin and Vold qualitatively explores the impact of an urban agriculture project on seven First Nation and non-First Nation young adults who engaged with the project as interns. ${ }^{8}$ The askîy project in Saskatoon, Saskatchewan, speaks to the current disconnect between the public and the mainstream food system, which is often far removed from consumers as they navigate grocery store aisles, kitchen cupboards, and restaurant menus. Agriculture projects in urban settings serve many purposes, with environmental, social, spiritual, agricultural, and food literacy implications, as the article explores. The integration of First Nations' knowledge and teachings makes this a particularly unique project, and the overall project provides an interesting template for how such programs can be integrated into local communities to reinforce involvement in food production and the overall food system, values related to local food and food sources, and attitudes towards healthy eating.

As Canada moves forward with implementing the various aspects of the Healthy Eating Strategy ${ }^{9}$ and A Food Policy for Canad $a,{ }^{10}$ both of which have highlighted the critical importance of healthy food policies to create supportive food environments and food systems, we should be mindful of the incredible potential for policies to shift consumer mindsets towards a new, healthier 'normal.' The articles in this issue emphasize the need for more policies, tools, programs, and practices that support food system reforms to provide nutrient-rich, healthier food options consistently across settings in Canada, and provide examples of local interventions that support environmental changes to increase access, availability, and affordability of healthy foods.

As we raise our expectations for healthy foods to be universally available, promoted, and appropriately priced, we will contribute to this generation and generations in the future-demanding, preferring, and consuming diets that promote health and wellness.

\section{Acknowledgements}

Dr. Lana Vanderlee is a Canadian Institutes of Health Research Banting Postdoctoral Fellow.

\section{References}

1. Garriguet D. Diet quality in Canada. Health Rep. 2009;20(3):41-52.

2. Hawkes C, Smith TG, Jewell J, et al. Smart food policies for obesity prevention. 2015. Lancet. 2015;385(9985): 2410-2421. doi: 10.1016/S0140-6736(14) 61745-1.

3. Birch LL. Development of food preferences. Annual review of nutrition. 1999;19(1):41-62.

4. The Office of the Chief Dental Officer of Canada. Commentary - What about the mouth? Connecting oral health and food environments. Health Promot Chronic Dis Prev Can. 2018; 38(1):3-5.

5. Raine KD, Atkey K, Lee Olstad D, et al. Healthy food procurement and nutrition standards in public facilities: evidence synthesis and consensus policy recommendations. Health Promot Chronic Dis Prev Can. 2018; 38(1):6-17.

6. McIsaac JLD, Jarvis SL, Spencer R, Kirk SFL. At-a-glance - "A tough sell”: findings from a qualitative analysis on the provision of healthy foods in recreation and sports settings. Health Promot Chronic Dis Prev Can. 2018; 38(1):18-22.

7. Coleman P, Gultig J, Emanuel B, Gee M, Orpana H. Status report - FoodReach Toronto: lowering food costs for social agencies and community groups. Health Promot Chronic Dis Prev Can. 2018;38(1):23-8.
8. Martin W, Vold L. Grow where you've never grown before! Health Promot Chronic Dis Prev Can. 2018;38(1): 29-35.

9. Health Canada. Health Canada's Healthy Eating Strategy. 2016. Available from: http://healthycanadians .gc.ca/publications/eating-nutrition /healthy-eating-strategy-canada -strategie-saine-alimentation/index -eng.php

10. Government of Canada. A Food Policy for Canada. 2017. Available from: https://www.canada.ca/en/campaign /food-policy.html 